\title{
Optimization of CT slice thickness in 3D-CRT and IMRT planning of low grade glioma
}

\author{
Z. Alirezaei ${ }^{1}$, A.R. Amouheidari², I. Abedi' ${ }^{1}$ F. Davanian³, P. Shokrani1 ${ }^{*}$, \\ M.R. Nazemzadeh4 \\ ${ }^{1}$ Department of Medical Physics, School of Medicine, Isfahan University of Medical Science, Isfahan, Iran \\ ${ }^{2}$ Department of Radiation Oncology, Isfahan Milad Hospital, Isfahan, Iran \\ ${ }^{3}$ Department of Radiology, Isfahan University of Medical Science, Isfahan, Iran \\ ${ }^{4}$ Research Center for Science and Technology in Medicine, Tehran University of Medical Sciences, Tehran, Iran
}

\begin{abstract}
- Original article

\section{*Corresponding authors: \\ Parvaneh Shokrani, PhD., \\ E-mail:}

shokrani@med.mui.ac.ir

Revised: March 2020

Accepted: April 2020

Int. J. Radiat. Res., April 2021; 19(2): 291-298

DOI: $10.29252 /$ ijrr.19.2.291

Background: This study aimed to select the optimum computerized (CT) slice thickness by analyzing its effect on the volumes and dosimetric parameters in treatment planning of low grade Glioma. Material and Methods: Fused brain CT and magnetic resonance imaging (MRI) images of 17 patients were used for treatment planning for three-dimensional conformal radiation therapy (3D -CRT) and Intensity-modulated radiation therapy (IMRT) using CT dataset with the thickness of $1.5 \mathrm{~mm}, 3,5$, and $10 \mathrm{~mm}$ slice. Volume of target and organs at risk (OARs), dosimetric parameters and contouring times using the reconstructed images were compared with the original dataset. Results: Using larger than $3 \mathrm{~mm}$ slice thicknesses resulted in significant increase up to $76 \%$ in target volumes as well as the volumes of OARs ( $p$-value $<0.05$ ). The variation in normalization point coordinates was also significant using larger than $3 \mathrm{~mm}$ slice thicknesses. The contouring time of tumor and OARs using the original data set was up to $35 \%$ more compared to the $3 \mathrm{~mm}$ dataset. Dosimetric parameters were comparable for 1.5 and $3 \mathrm{~mm}$ datasets, however, significant difference of up to $200 \%$ was observed for 5 and $10 \mathrm{~mm}$ datasets $(\mathrm{P}<0.05)$. Conclusions: CT slices larger than $3 \mathrm{~mm}$ resulted in significant inaccuracies in volumes and dose coverage of target and OARs. Although using the slice thickness larger than $1.5 \mathrm{~mm}$ reduced contouring time significantly, this slice thickness is only recommended when the oncologist is not concerned about the dose received by the small critical organs especially those adjacent to the tumor.
\end{abstract}

Keywords: Conformity index, Homogeneity Index, Low grade glioma, Brain tumors, CT slice thickness, Radiotherapy.

\section{INTRODUCTION}

Low-grade Gliomas (LGGs) account for about two-thirds of CNS tumors in adolescents and young adults in the age group 20-44 years $(1,2)$. With a relatively long survival of about 6 years, life quality of these patients after radiotherapy is influenced by the maximum dose provided to the tumor and the degree of normal brain tissue sparing (3-8).

CT and MR Imaging are the modalities of choice for tumor and organs at risk (OARs) volume delineation in radiotherapy treatment planning (RTP) of brain tumors $(7,8)$. Using fused CT/MR images, a combination of the following characteristics is utilized in three dimensional 
conformal radiation therapy (3D-CRT) or IMRT (Intensity Modulate Radiation Therapy) of brain tumors: CT number information for density correction in the dose calculations, CT image of cortical bone for RT set up verification and MR low contrast detectability for determining the gross or clinical tumor volumes $(9,10)$. However, the accuracy of target and OAR volume determination and therefore accuracy of dose determining results is challenged by the impact of CT image slice thickness and MR inherent geometric image fidelity (11-14).

Determining the optimal slice thickness depends on the size, shape and volume of the target and OARs. For 3DRTP of brain tumors, 4 $\mathrm{mm}$ and 8-10 $\mathrm{mm}$ slice thicknesses have been suggested as optimum for targets with diameters of $1.5-3 \mathrm{~cm}$ and diameters larger than $4 \mathrm{~cm}$, respectively (11-14). However, when using co-registered/fused MR/CT images, larger slice thicknesses have been suggested (15). Phantom studies using spherical volumes have used the smallest available slice thickness for targets with diameters less than $1.5 \mathrm{~cm}$ (14). In general, in RTP, the delineated volume may increase (11-14) or decrease (16) with increase in the CT slice thickness, especially for smaller volumes (12). The inaccuracy of volume delineation in RTP can introduce significant error in the dose calculation results including (14) mean dose of planning target volume (PTV), tumor control probability (TCP) as well as in dose distribution analysis tools like homogeneity Index (HI) and dose conformity index (CI) (11).

Since the definition of the volumes in RTP depends very strongly on the slice thickness, the optimal slice thickness should be selected based on the tumor size and the structure of the OARs such as the optical structure, brain stem and other normal structures (11-14).

In this clinical study, the goal was to assess the effect of CT slice thickness on organs volumes and calculated target and dosimetric parameters in treating plan of low grade glioma patients on The results of this study were applied towards optimizing our routine imaging protocols and treatment planning procedure in order to achieve the tradeoff between the accuracy and the time dedicated to contouring of target volumes and OARs of LGG patients. This research made a step forward in the upgrade of IMRT treatment planning procedure of glioma patients in our center.

\section{MATERIALS AND METHODS}

\section{Patient's selection}

CT and MRI images of 17 patients who were being treated with radiotherapy for low grade Glioma tumors were used, after obtaining institutional review board approval (IR.mui.rec.1397.3.153, with date of issue 03/1397) and informed written consents from all participants. Table 1 represents the patient's demographics briefly.

Table 1. Patients' demographics.

\begin{tabular}{|c|c|}
\hline Age Range (y) & 19-54 \\
\hline Gender & Memale: 10 \\
Male: 7
\end{tabular}

\section{Simulation}

For planning, contrast-enhanced CT images with $1.5 \mathrm{~mm}$ slice thickness were obtained 4-6 weeks after surgery or biopsy using a Sensation 64 CT unit (Siemens Healthcare, Erlangen, Germany), while utilizing neck and head thermoplastic masks patients were immobilized. Gadolinium-improved T1-weighted and T2weighted MR images were acquired using a 1.5 Tesla Magnetom Aera MRI Scanner (Siemens Healthcare, Erlangen, Germany). These CT and MR images were co-registered to make the fused images according to the International Commission on Radiation Units and

Int. J. Radiat. Res., Vol. 19 No. 2, April 2021 
Measurements report 62 (ICRU-62)(17). A visual check was carried out at the registration procedure end and the oncologist co-registered images were edited manually by the radiation when the satisfactory results were obtained. Patient image datasets of $1.5 \mathrm{~mm}$ slice thickness (the original dataset) were then rebuilt with different slice thickness of 3, 5, and $10 \mathrm{~mm}$ (the reconstructed data set). The data set with 10 $\mathrm{mm}$ slice thickness was used for completeness and was not intended for clinical application.

\section{Contouring}

Contouring of target and OAR volumes was performed by a radiation oncologist on CT images and CT- MRI fused images were used for diagnostic purposes. Gross tumor volume (GTV) was explained as the operating cavity with any residual contrast-improving tissue on T1-weighted magnetic resonance imaging or as the residual enhanced tumor. The clinical target volume (CTV) was determined using a $2 \mathrm{~cm}$ margin around the GTV to contain any potential subclinical tumor, with an attempt to consider the potential OARs and natural anatomic boundaries. A primary planning target volume (PTV) was created by addition of a $5.0-\mathrm{mm}$ margin to comprise arrangement uncertainties. A smaller and second PTV, denoted as the boost PTV, was created by addition of $5.0-\mathrm{mm}$ margin to the GTV (18). For each patient, the normal tissues contoured involved the optic nerves, optic chiasma and retina, brain stem and corpus callosum. The tumor and OAR volumes were delineated on the original $(1.5-\mathrm{mm}$ slice thickness) as well as the reconstructed (3, 5 and $10 \mathrm{~mm}$ slice thicknesses) datasets.

\section{Phantom study}

A simple acrylic phantom (density $=1.20 \mathrm{~g} /$ $\mathrm{cm}^{3}$ ) was made, consisting of 5 layers of $2.2 \mathrm{~cm}$ thickness. In each layer a 2 dimensional array of cylindrical holes (diameter $=3.75 \mathrm{~cm}$ volume $=71.2 \mathrm{cc}$ ) was machined. The cylindrical holes were filled with melted paraffin (density $=0.93$ $\mathrm{g} / \mathrm{cm} 3)$. The layers were then placed and fixed on top of each other, making a $170 \times 110 \times 120$ $\mathrm{cm}^{3}$ block (figure 1.A). The imaging procedures were carried out using the same CT and MR units and scan protocols that were used for the clinical study (figure 1. B and C). The geometric accuracy of CT and MR images was analyzed by comparing the diameter and volume of holes in the original and the reconstructed data sets and the phantom itself.

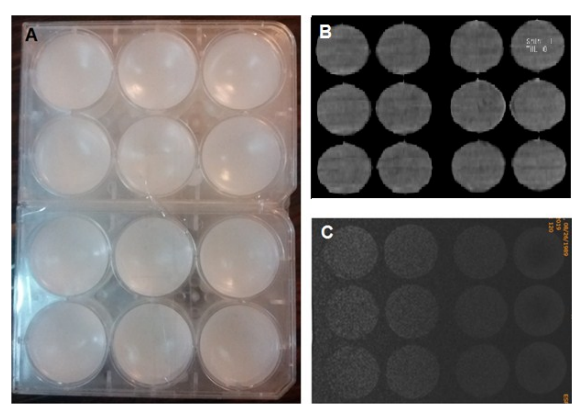

Figure 1. A The Photo of the acrylic phantom containing arrays of paraffin filled cylindrical holes, B The CT and C the T1 -weighted $M R$ images of the phantom using a repetition time (TR) of $350 \mathrm{~ms}$ and echo time (TE) of $10 \mathrm{~ms}$.

\section{Treatment Planning}

IMRT and 3D-CRT plans were designed for each patient utilizing the original and reconstructed images of $1.5,3,5$, and $10 \mathrm{~mm} \mathrm{CT}$ slice thicknesses. A radiation oncologist performed delineations of target and organs at risk (OAR). A Prowess Panther planning system version 5.5 (Prowess Inc., Concord, CA) which was commissioned and verified for the $6 \mathrm{MV}$ and $15 \mathrm{MV}$ photon beams of an ONCOR linear accelerators (linac), (Siemens Healthcare, Erlangen, Germany) was used for both 3D-CRT forward and IMRT inverse plans. The linac was armed with multi-leaf collimators (MLC) for step -and-shoot IMRT delivery. A 6 MV or a combination of $15 \mathrm{MV}$ and $6 \mathrm{MV}$ were utilized for 3D-CRT plans according to the location and size of the PTV, the required dose uniformity and organs at risk dose specifications. The prescription dose for 3D-CRT was 45.0 Gy to the PTV and an additional dose of 9.0 Gy to boost the total PTV dose to 54.0 Gy. 3D-CRT plans included two opposed fields $(90$ and 270 degree) and one vertex field with or without a 15-degree wedge. The angel of gantry, collimator and couch varied between $0.0,40.0$ to 50 and 270.0 degrees, respectively. IMRT plans using 6 MV photons included five fields each comprised of five segments. The angels of beams were 
optimized automatically according to the location and size of the PTV, the required dose uniformity and organs at risk dose constraints as presented by the Quantitative Analyses of Normal Tissue Effects in the Clinic (QUANTEC) (14). For all 3DCRT and IMRT plans, the dose volume histograms (DVH) were computed for the OARs and PTV. Specific dose points included the maximum dose $\left(D_{\max }\right)$, minimum dose $\left(D_{\min }\right)$, and mean dose ( $D_{\text {mean }}$ ) to the PTV and OARs were analyzed. By comparing the plans, designed using CT images of different thicknesses, the effect of slice thickness on delineated volume size and calculated DVHs of target and OARs was analyzed. In addition, ICRU83 dose-volume points such as D2\%, D50\%, D98\% were calculated to define the homogeneity index (HI) by using equation 1 (19):

$$
H I=\frac{\left(D_{2 \%}-D_{98 \%}\right)}{D_{50 \%}}
$$

Conformity index (CI) was determined as well utilizing tissue volume receiving $95 \%$ dose using equation 2 (20):

$$
C I_{95 \%}=\frac{\text { Tissue volume receiving the } 95 \% \text { dose }}{\text { PTV Volume }}
$$

The shift in coordinates of the normalization point, i. e. the point that isodose lines were normalized to a stated point other than the isocenter (probably the hot spot) (19), were calculated for each plan using the different CT image data sets and with respect to a fixed reference point. The $\mathrm{x}, \mathrm{y}$ and $\mathrm{z}$ direction is corresponding to right-left, anterior-posterior, and superior-inferior, respectively. The average time dedicated to contouring of target by the radiation oncologist and also contouring of OARs by a physicist using original and reconstructed CT datasets was recorded.

\section{Statistical Analysis}

An independent sample t-test (SPSS 20) was used to examine the association between the changes in calculated parameters with CT slice thicknesses variation for each plan. A test result with $\mathrm{p} \leq 0.05$ was regarded as statistically significant.

\section{RESULTS}

\section{Volume Analysis \\ Phantom study}

Findings of the phantom study indicated that in both CT and MRI images, increasing slice thickness from 1.5 to $3 \mathrm{~mm}$ resulted in insignificant increase on the imaged volume sizes. In contrast, using 5 and $10 \mathrm{~mm}$ slices for $3 \mathrm{D}$ volume reconstruction, resulted in significant increase of $12 \%$ and up to $37 \%$ in imaged volume sizes, respectively.

\section{Patient study}

Figure 2 shows that the volume of target is increased with increase in slice thickness. The calculated data from patients showed that changing the slice thickness from 1.5 to $3 \mathrm{~mm}$ did not result in significant change in volume $(\Delta \mathrm{V}=12 \%$; P-value $>0.05)$. However, the result revealed significant change in the target volume when $5 \mathrm{~mm}(\Delta \mathrm{V}=47 \%$; $\mathrm{P}$-value $<0.05)$ and $10 \mathrm{~mm}$ $(\Delta \mathrm{V}=77 \% ; \quad \mathrm{P}$-value $<0.05)$ reconstructed sets were used compared to the original dataset. Contouring with 3-mm data set instead of the original data set, resulted in significant increase only in the volume of body of corpus callosum $(18 \%)$ and left optic nerve (12\%). Using larger than $3 \mathrm{~mm}$ slice thicknesses resulted in significant increase in volume of retina, chiasma, genu and splenium as well as corpus callosum and left optic nerve figure 3 .

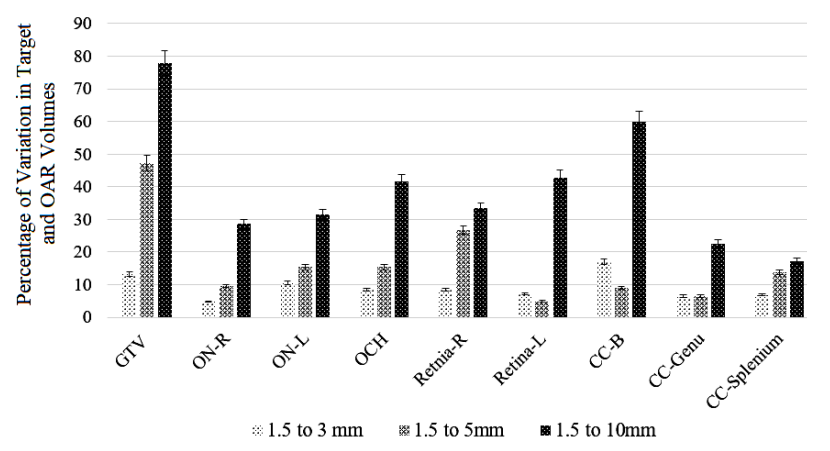

Figure 2. Relative variation (\%) of target and OAR volumes delineated using $3 \mathrm{~mm}, 5 \mathrm{~mm}$ and $10 \mathrm{~mm}$ reconstructed dataset compared to $1.5 \mathrm{~mm}$ dataset. Increase in slice thickness resulted in increase in volumes of both target and OARs. Abbreviations: R: Right; L: Left; ON: Optic Nerve; OCH: Optic Chiasma; CC-B: Corpus callosum Body; CC-Genu: Genu of Corpus Callosum; CC-Splenium: Splenium of Corpus Callosum. 


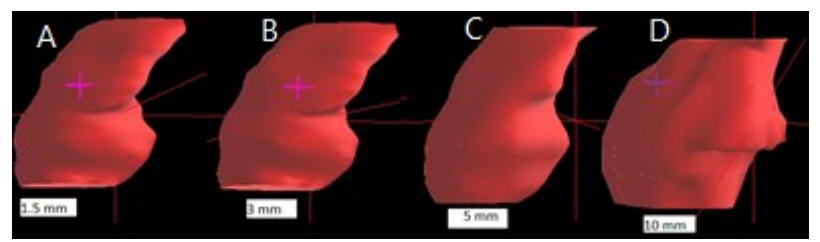

Figure 3. An example of a 3D reconstructed tumor volume using, A: $1.5 \mathrm{~mm}$ slice, B: $3 \mathrm{~mm}$ slice, C: $5 \mathrm{~mm}$ slice and D: 10 $\mathrm{mm}$ slice datasets shows an increase in slice thickness resulted in increase in tumor volume.

\section{CI and HI Variation}

CI decreased when using larger CT slice thicknesses. For 3D-CRT, the change in $\mathrm{CI}(\Delta \mathrm{CI})$ was significant when using 5 and $10 \mathrm{~mm}$ reconstructed data sets compared to $\mathrm{CI}$ for the original data set where $\mathrm{CI}=0.69: \Delta \mathrm{CI}=-0.03$, -0.11 and -0.13 for 3,5 and $10 \mathrm{~mm}$ data sets, respectively. In IMRT plans, also, $\Delta \mathrm{CI}$ was significant for 5 and $10 \mathrm{~mm}$ reconstructed data sets compared to $\mathrm{CI}$ for the original data set where $\mathrm{CI}=0.78$ : $\Delta \mathrm{CI}=0.05,0.08$ and 0.15 for $3 \mathrm{~mm}, 5 \mathrm{~mm}$ and $10 \mathrm{~mm}$ data sets, respectively. Change in $\mathrm{HI}(\Delta \mathrm{HI})$ was not significant by changing slice thickness in 3D-CRT. In IMRT, however, $\mathrm{HI}$ increased from 0.11 for the original data set to 0.13 and 0.15 for 5 and $10 \mathrm{~mm}$ data sets, respectively (P-value $<0.05$ ).

\section{Normalization point shift in 3D-CRT plans}

In the 3D-CRT treatment plans, the average shift in normalization point coordinates in the $\mathrm{x}$ direction was only significant for the $10 \mathrm{~mm}$ data set $(\Delta x=0.3 \mathrm{~cm})$, (P-value $=0.02)$. In $\mathrm{y}$ and $\mathrm{z}$ directions, significant changes (in $\mathrm{cm}$ ) were as follows: for $3 \mathrm{~mm}, 5 \mathrm{~mm}$ and $10 \mathrm{~mm}$ data sets $\Delta \mathrm{y}=0.3 \mathrm{~cm}, \Delta \mathrm{z}=0.2 \mathrm{~cm} ; \Delta \mathrm{y}=0.7 \mathrm{~cm}, \Delta \mathrm{z}=0.4 \mathrm{~cm}$ and $\Delta \mathrm{y}=1.1 \mathrm{~cm}, \Delta \mathrm{z}=0.8 \mathrm{~cm}, \quad$ respectively $(\mathrm{P}$-value $=0.03$ ).

\section{Contouring time evaluation}

The average time dedicated to delineate the target and OARs using the original data set compared to the reconstructed data set was considerably higher. Using the original data sets, to contour the target, it took up to 32,52 and 64 $\%$ longer and to contour the OARs, it took up to
35, 51 and $66 \%$ longer than using the slice thickness of 3, 5 and $10 \mathrm{~mm}$, respectively.

\section{Dosimetric parameters analysis}

Figure 4 shows the slice thickness's impacts on target dosimetry calculation results. For both 3D-CRT and IMRT techniques, $\mathrm{D}_{\text {min, }} \mathrm{D}_{\text {mean }}$ and $D_{\max }$ decreased by changing the slice thickness from $1.5 \mathrm{~mm}$ to $3 \mathrm{~mm}$ (P-value> 0.05), from $1.5 \mathrm{~mm}$ to $5-\mathrm{mm}(\mathrm{P}<0.05)$, and from 1.5 to $10 \mathrm{~mm}$ $(\mathrm{P}<0.05)$. A decrease of up to $10 \%$ and $14 \%$ in target maximum dose was seen in both IMRT and 3D-CRT techniques using 5 and $10 \mathrm{~mm}$ slice thickness instead of $1.5 \mathrm{~mm}$.

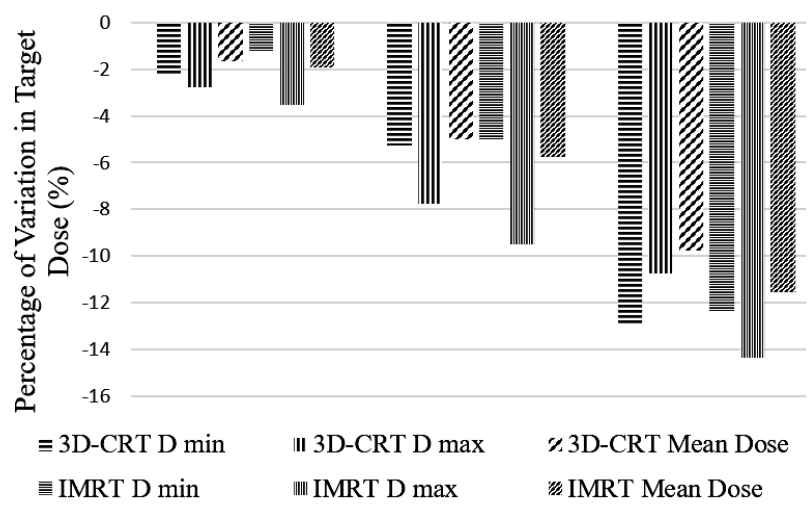

Figure 4. Relative decrease in dosimetric parameters (Dmin, Dmean and Dmax) for target volume (CTV) using reconstructed CT slice thicknesses of 3,5 and $10 \mathrm{~mm}$ in 3D-CRT and IMRT plans compared to the original data set $(1.5 \mathrm{~mm})$

For both techniques, Dmax for all of the OARs increased by increasing the slice thickness (figure 5). The increase in Dmax was significant for the left optic nerve (22\%) and corpus callusom $(25 \%)$ by changing the thickness of the slices from $1.5 \mathrm{~mm}$ to $3 \mathrm{~mm}$ in both techniques (P-value). Increasing slice thickness from 1.5 to 5 or $10 \mathrm{~mm}$ in both 3D-CRT and IMRT, showed significant increase in all dosimetric parameters for all OARs (P-value <0.05). The maximum Dmax change of $95 \%$ for the left optic nerve and $200 \%$ for the left retina was recorded when changing the slice thickness to $5 \mathrm{~mm}$ and 10 $\mathrm{mm}$, respectively. 


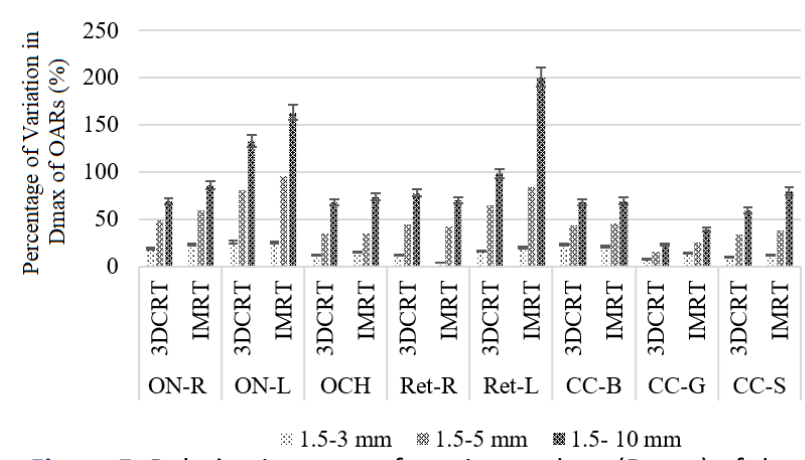

Figure 5. Relative increase of maximum dose (Dmax) of the optic nerve, chiasma, retina, and corpus callusom (body, genu, and splenium) in 3D-CRT and IMRT techniques, using reconstructed data sets of 3,5 and $10 \mathrm{~mm}$ compared to the original data set of $1.5 \mathrm{~mm}$ thickness. Abbreviations: R: Right; L: Left; ON: Optic Nerve; OCH: Optic Chiasma; BS: Brain Stem; CC-B: Body of Corpus Callusom; CC-Genu: Genu of Corpus Callusom; CC-Splenium: Splenium of Corpus Callusom.

\section{DISCUSSION}

In radiation treatment planning, patient CT images are used for tumor and OAR volume delineations. Therefore, accuracy of dose calculations can be affected if quality of CT images is not adequate. Among other image properties, selecting the optimum slice thickness becomes important in order to maintain a balance between contouring accuracy and time involved in volume delineations by the physician (11-15,17). This work was aimed to find the optimum CT image slice thickness for radiation treating plan LGG patients in our radiotherapy department.

Significant increase in the calculated volumes of the holes in phantom compared to the actual volumes, showed that $3 \mathrm{~mm}$ slice thickness is a reasonable choice to contour targets in the range of 3-4 cm in diameter which is in good agreement with other phantom studies (11-14). However, in contrast to other phantom studies in which using 8-10 $\mathrm{mm}$ slice thickness were recommended in contouring volumes larger than $25 \mathrm{cc}$, our study does not recommend using slice thickness more than $3 \mathrm{~mm}$ even for the volumes in the range of $75 \mathrm{cc}$ (11-14).

In the clinical study, an overall significant increase in target volume with increasing the slice thickness was in agreement with the results reported by Srivastava et al. (14). In contrast to our results, there has been reports indicating decrease of target volumes when using larger slice thicknesses $(11,13)$. This could be due to missing a part of the target in contouring of images of larger slice thicknesses. Another potential reason for this difference is when some of the slices in the original data set are selectively removed (11), instead of using reconstructed images of larger thicknesses in contouring.

Although changing slice thickness from 1.5 $\mathrm{mm}$ to $3 \mathrm{~mm}$ resulted in significant increase only in the volume of body of corpus callosum and left optic nerve, using slice thickness larger than $3 \mathrm{~mm}$ resulted in significant increase in the volume of all OARs including left and right optic nerve, retina, chiasma, corpus callosum, genu and splenium. Other studies suggested to use images with slice thicknesses of 4 or $8 \mathrm{~mm}$ for contouring in 3D-CRT treatment planning, provided that the image has sufficient contrast (15). Our results showed that using slice thickness more than $3 \mathrm{~mm}$ is not reasonable for contouring in treatment planning. As a consequence, finding our study does not confirm this recommendation. Prabhakar et al. also showed that, the $2.5 \mathrm{~mm}$ and $5 \mathrm{~mm}$ slice thickness is sufficient for the target volumes ranging from 25-220cc (13). In contrast to the study of Prabhakar et al., our results showed using $5 \mathrm{~mm}$ slice thickness resulted in significant increase in the target volume and is not recommended to contour volumes in the range of $85 \mathrm{cc}$.

$\mathrm{CI}$, a key parameter for assesing a treatment plan quality was also evaluated using different CT data sets. CI presents a measure of dose to normal tissue related to PTV coverage, and the closer $\mathrm{CI}$ is to unity, the more conformal the plan is $(19,24)$. In this study, using larger slice thicknesses caused a significant reduction in $\mathrm{CI}$ in both 3D-CRT and IMRT techniques, similar to the results reported in other studies $(11,14)$. Based on the explanation, $\mathrm{HI}=0$ shows the most hemogenous treatment plan. In general, a lower $\mathrm{HI}$ value is preffred. Although HI increased with incresing slice thickness, the variation was only statistically significant for IMRT plans, using 3 $\mathrm{mm}$ and $5 \mathrm{~mm}$ data sets. The largest change in

Int. J. Radiat. Res., Vol. 19 No. 1, April 2021 
HI was due to using slices thickness of $10 \mathrm{~mm}$ in IMRT plans which was in a good agreemant with other studies $(13,14)$. Therefore, it should be concluded that for small targets, such as those seen in LGG patients and also targetrs treated using IMRT, the smaller slice thickness shuold be chosed.

Variation in normalization point due to change in delineated volume size can affect the treatment outcome. The reason is that in 3D-CRT plans, $1 \mathrm{~cm}$ margin around CTV may not be adequate to compensate the shift in normalization point. As a result, target under dosage with an increase in normal tissue dose may occur, especially when sensitive structures are adjacent to the target $[11,13]$. Our results revealed that using image datasets of larger than $1.5 \mathrm{~mm}$ slice thickness resulted in the normalization point shift, in treatment plan of all patients. The maximum shift occurred in the $Y$ direction (Anterior Posterior) when slice thickness increased. Similar to other studies (11, 13), the maximum shit in normalization point in the $Y$ direction may be due to the direction of tumor extension in this direction.

A significant decrease in all dosimetric parameters for target was revealed using $5 \mathrm{~mm}$ and $10 \mathrm{~mm}$ slice thickness, which resulted in a significant target underdoseage. In a similar study (13), it was reported that incresing the slice thickness from $2.5 \mathrm{~mm}$ to 5 and $10 \mathrm{~mm}$ resulted in the tumor underdosage, specifically for horseshoe-shaped targets, and up to $5 \%$ decrease in IMRT mean target dose for $5 \mathrm{~mm}$ slice thickness compared to $1 \mathrm{~mm}$.

The highest dose recieved by the OARs increased using larger slice thicknesses in both IMRT and 3D-CRT plans. This increase was only significant for optic nerve in both techniques and corpus callosum in 3D-CRT technique, using slice thickness of larger than $1.5 \mathrm{~mm}$ to $3 \mathrm{~mm}$. Since small structures like the optic nerves and retina are involved in treatment plan of LGG, estimating the OAR volumes may encounter considerable clinical implications, resulting in a compromise between the recommended dose-volume criteria $(12,14)$ and reflecting the real clinical significance. This research showed that the choice of optimal slice thickness is of great importance in sparing these small structures, especially for the optic nerve due to its critical location. Choosing an inappropriate slice thickness for the contouring of the optic nerve may result in miscalculation of dose delivered to optic nerve, as emphasized in other studies (14, 20-22).

Despite the small volume of some organs, specifically the retina, there was no significant difference between the calculated volume using axial images and the dosimetric parameters with an alteration in slice thickness from 1.5 to $3 \mathrm{~mm}$. Nevertheless, due to the small size of the retina, it has been recommended to use the smallest slice thickness available for its contouring $(13,14)$. Therefore, we cannot conclusively specify that images with a slice thickness of $1.5 \mathrm{~mm}$ can be replaced by images with a thickness of $3 \mathrm{~mm}$ for this organ and organs of similar size. Several factors such as proximity to the tumor may have significant effect on this issue. The drawback of choosing 1 or $1.5 \mathrm{~mm}$ slice thickness, in order to obtain better dosimetric results, can be overheating of CT scanner X-ray tube as well as longer contouring times oncologist spent on contouring the target and OARS.

\section{CONCLUSION}

In conclusion, results showed significant inaccuracies for both target and OARs, when contouring was done using reconstructed $3 \mathrm{~mm}$ CT data sets. Although using the $3 \mathrm{~mm}$ instead of the $1.5 \mathrm{~mm}$ data sets reduced contouring time significantly, care should be taken if the margin around CTV is not adequate to compensate the shift of normalization point. Moreover, this slice thickness is only recommended when the oncologist is not concerned about the dose received by the small critical organs, especially those located adjacent to the target volume.

Conflicts of interest: Declared none.

\section{REFERENCES}

1. Taphoorn M, Schiphorst AK, Snoek F, Lindeboom J, Wolbers J, Karim A, Huijgens P, Heimans J (1994) Cognitive 


\section{Alirezaei et al. / Optimization of CT Slice thickness in 3D-CRT and IMRT planning}

functions and quality of life in patients with low-grade gliomas: the impact of radiotherapy. Annals of Neurology: Official Journal of the American Neurological Association and the Child Neurology Society, 36(1): 48-54.

2. Claus EB, Horlacher A, Hsu L, Schwartz RB, Dello-lacono D, Talos F, Jolesz FA, Black PM (2005) Survival rates in patients with low-grade glioma after intraoperative magnetic resonance image guidance. Cancer: Interdisciplinary International Journal of the American Cancer Society, 103(6): 1227-1233.

3. Berger MS, Deliganis AV, Dobbins J, Keles GE (1994) The effect of extent of resection on recurrence in patients with low grade cerebral hemisphere gliomas. Cancer, 74(6): 1784-1791.

4. Klein $M$, Heimans J, Aaronson N, Van der Ploeg H, Grit J, Muller M, Postma T, Mooij J, Boerman R, Beute G (2002) Effect of radiotherapy and other treatment-related factors on mid-term to long-term cognitive sequelae in low-grade gliomas: a comparative study. The Lancet, 360(9343): 1361-1368.

5. Shaw $E$, Arusell R, Scheithauer B, O'fallon J, O'neill B, Dinapoli R, Nelson D, Earle J, Jones C, Cascino T (2002) Prospective randomized trial of low-versus high-dose radiation therapy in adults with supratentorial low-grade glioma: initial report of a North Central Cancer Treatment Group/Radiation Therapy Oncology Group/Eastern Cooperative Oncology Group study. J Clin Oncol, 20(9): 22672276.

6. Surma-aho $O$, Niemelä $M$, Vilkki J, Kouri $M$, Brander $A$, Salonen O, Paetau A, Kallio M, J Pyykkönen L, Jääskeläinen $J$ (2001) Adverse long-term effects of brain radiotherapy in adult low-grade glioma patients. Neurology, 56(10): 1285 1290.

7. Van den Bent $M$, Afra $D$, De Witte $O$, Hassel $M B$, Schraub $S$, Hoang-Xuan K, Malmström P, Collette L, Piérart M, Mirimanoff $R$ (2005) Long-term efficacy of early versus delayed radiotherapy for low-grade astrocytoma and oligodendroglioma in adults: the EORTC 22845 randomised trial. The Lancet, 366(9490): 985-990.

8. Pardo FS, Aronen HJ, Kennedy D, Moulton G, Paiva K, Okunieff $P$, Schmidt EV, Hochberg FH, Harsh GR, Fischman AJ (1994) Functional cerebral imaging in the evaluation and radiotherapeutic treatment planning of patients with malignant glioma. Int J Radiat Oncol Biol Phys, 30(3): 663669.

9. Veninga $T$, Huisman $H$, van der Maazen RW, Huizenga $H$ (2004) Clinical validation of the normalized mutual information method for registration of CT and MR images in radiotherapy of brain tumors. J Appl Clin Med Phys, 5(3): 66-79.

10. Webster GJ, Kilgallon J, Ho KF, Rowbottom CG, Slevin NJ, Mackay RI (2009) A novel imaging technique for fusion of high-quality immobilised MR images of the head and neck with CT scans for radiotherapy target delineation. The British Journal of Radiology, 82(978): 497-503.

11. Caivano R, Fiorentino A, Pedicini P, Califano G, Fusco V (2014) The impact of computed tomography slice thickness on the assessment of stereotactic, 3D conformal and intensity-modulated radiotherapy of brain tumors. Clinical and Translational Oncology, 16(5): 503-508.

12. Fiorentino A, Pedicini P, Caivano R, Fusco V (2013) What is the best way to evaluate clinical target volume for radiotherapy of brain tumors? CNS Oncology, 2(6): 475-477.

13. Prabhakar R, Ganesh T, Rath GK, Julka PK, Sridhar PS, Joshi RC, Thulkar S (2009) Impact of different CT slice thickness on clinical target volume for 3D conformal radiation therapy. Med Dosim, 34(1): 36-41.

14. Srivastava S, Cheng C-W, Das I (2016) The effect of slice thickness on target and organs at risk volumes, dosimetric coverage and radiobiological impact in IMRT planning. Clinical and Translational Oncology, 18(5): 469-479.

15. Somigliana A, Zonca G, Loi G, Sichirollo AE (1996) How thick should $\mathrm{CT} / \mathrm{MR}$ slices be to plan conformal radiotherapy? A study on the accuracy of three-dimensional volume reconstruction. Tumori Journal, 82(5): 470-472.

16. Prionas ND, Ray S, Boone JM (2010) Volume assessment accuracy in computed tomography: a phantom study. J Appl Clin Med Phys, 11(2): 168-180.

17. Chavaudra J, Bridier A (2001) Definition of volumes in external radiotherapy: ICRU reports 50 and 62 . Cancer radiotherapie: Journal de la Societe Francaise de Radiotherapie Oncologique, 5(5): 472-478.

18. Kantor G, Loiseau H, Vital A, Mazeron J (2001) Gross tumor volume (GTV) and clinical target volume (CTV) in adult gliomas. Cancer radiotherapie: journal de la Societe francaise de radiotherapie oncologique, 5(5): 571-580.

19. Pickett B, Altieri G (1992) Normalization: What does it really mean? Med Dosim, 17(1): 15-27.

20. Ahnesjö A, Aspradakis MM (1999) Dose calculations for external photon beams in radiotherapy. Phys Med Biol, 44 (11): $R 99$.

21. Cox JD, Stetz J, Pajak TF (1995) Toxicity criteria of the radiation therapy oncology group (RTOG) and the European organization for research and treatment of cancer (EORTC). Int J Radiat Oncol Biol Phys, 31(5): 1341-1346.

22. Musat $E$, Roelofs $E$, Bar-Deroma R, Fenton $P$, Gulyban $A$, Collette L, Stupp R, Weber DC, Davis JB, Aird E (2010) Dummy run and conformity indices in the ongoing EORTC low-grade glioma trial 22033-26033: first evaluation of quality of radiotherapy planning. Radiother Oncol, 95(2): 218-224.

Int. J. Radiat. Res., Vol. 19 No. 2, April 2021 